Ferrata Storti Foundation

\title{
uPA-mediated plasminogen activation is enhanced by polyphosphate
}

Claire S. Whyte and Nicola J. Mutch

Aberdeen Cardiovascular \& Diabetes Center, School of Medicine, Medical Sciences \& Nutrition, Institute of Medical Sciences, University of Aberdeen, Aberdeen, UK

\section{ABSTRACT}

Haematologica 2021

Volume 106(2):522-531

\section{Correspondence:}

NICOLA J. MUTCH

n.j.mutch@abdn.ac.uk

Received: September 12, 2019.

Accepted: January 31, 2020.

Pre-published: February 6, 2020.

https://doi.org/10.3324/haematol.2019.237966

(C)2021 Ferrata Storti Foundation

Material published in Haematologica is covered by copyright. All rights are reserved to the Ferrata Storti Foundation. Use of published material is allowed under the following terms and conditions:

https://creativecommons.org/licenses/by-nc/4.0/legalcode. Copies of published material are allowed for personal or internal use. Sharing published material for non-commercial purposes is subject to the following conditions:

https://creativecommons.org/licenses/by-nc/4.0/legalcode, sect. 3. Reproducing and sharing published material for commercial purposes is not allowed without permission in writing from the publisher. issue plasminogen activator (tPA) and urokinase plasminogen activator (uPA) differ in their modes of action. Efficient tPA-mediated plasminogen activation requires binding to fibrin. In contrast, uPA is fibrin independent and activates plasminogen in solution or when associated with its cellular receptor urokinase protease activated receptor (uPAR). We have previously shown that polyphosphate (polyP), alters fibrin structure and attenuates tPA and plasminogen binding to fibrin, thereby down-regulating fibrinolysis. Here we investigate the impact of polyP on uPA-mediated fibrinolysis. As previously reported polyP of an average chain length of 65 (poly $\mathrm{P}_{6}$ ) delays tPA-mediated fibrinolysis. The rate of plasmin generation was also delayed and reduced 1.6-fold in poly $\mathrm{P}_{\text {ws }}$-containing clots $(0.74 \pm 0.06$ vs. $1.17 \pm 0.14 \mathrm{pM} / \mathrm{s}$ in $P<0.05)$. Analysis of tPA-mediated fibrinolysis in real-time by confocal microscopy was significantly slower in poly $\mathrm{P}_{\omega s}$-containing clots. In marked contrast, poly $\mathrm{P}_{\omega}$ augmented the rate of uPA-mediated plasmin generation 4.7 -fold $(3.96 \pm 0.34$ vs. $0.84 \pm 0.08 \mathrm{pM} / \mathrm{s} ; P<0.001)$ and acce-lerated fibrinolysis ( $\mathrm{t} 1 / 264.5 \pm 1.7 \mathrm{~min} v s .108 .2 \pm 3.8 \mathrm{~min} ; P<0.001)$. Analysis of lysis in real-time confirmed that polyP $\mathrm{P}_{6}$ enhanced uPA-mediated fibrino-lysis. Varying the plasminogen concentration $(0.125-1 \mu \mathrm{M})$ in clots dose-dependently enhanced uPA-mediated fibrinolysis, while negligible changes were observed on tPA-mediated fibrinolysis. The accelerating effect of poly $\mathrm{P}_{\sigma}$ on uPA-mediated fibrinolysis was overcome by additional plasminogen, while the down-regulation of tPA-mediated lysis and plasmin generation was largely unaffected. poly $\mathrm{P}_{6}$ exerts opposing effects on tPA- and uPA-mediated fibrinolysis, attenuating the fibrin cofactor function in tPA-media-ted plasminogen activation. In contrast, polyP may facilitate the interaction between fibrin-independent uPA and plasminogen thereby accelerating plasmin generation and downstream fibrinolysis.

\section{Introduction}

Polyphosphate (polyP) is a biomolecule composed of orthophosphate residues (Pi) linked by phosphoanhydride bonds. ${ }^{1}$ polyP of average chain length of 60-100-mers is a constituent of platelet dense granules and is released following stimulation of platelets with different agonists. ${ }^{1,2}$ polyP acts at numerous points in the coagulation cascade to augment clot formation, including stimulating factor XII (FXII) activation, thrombin-mediated factor XI (FXI) activation and interfering with the function of TFPI. ${ }^{3}$ Our work has shown that polyP interferes with fibrin polymerization by stunting protofibril growth, producing a heterogeneous network of dense 'knotted' regions interspersed by pores with altered mechanical properties. ${ }^{4}$

Plasmin is the serine protease responsible for degradation of fibrin. Two forms of the zymogen precursor plasminogen circulate in plasma, the native more abundant form, Glu-plasminogen, and the intermediate form Lys-plasminogen, formed by cleavage of the $\mathrm{N}$-terminal peptide from Glu-plasminogen. ${ }^{5}$ Lys-plasminogen exists in flexible open conformation, with an approximately 10-fold higher binding affinity for plasminogen activators thereby facilitating its activation. ${ }^{6.9}$ Two-chain plasmin is formed by enzymatic cleavage of plasminogen at Arg561-Val562., ${ }^{5,10}$ The two 
main physiological plasminogen activators are the serine proteases tissue plasminogen activator (tPA) and urokinase plasminogen activator (uPA). Efficient plasminogen activation by tPA requires fibrin as a cofactor, acting as a template for its own dissolution by binding tPA and plasminogen. ${ }^{11}$ uPA is fibrin-independent and efficiently activates plasminogen in solution, but is often found in association with its cellular receptor, urokinase protease activated receptor (uPAR). ${ }^{12}$ uPAR does not have a catalytic role but acts to localize plasminogen and uPA to the cell surface increasing local reactant concentration.

We have previously shown that binding of tPA and plasminogen to fibrin is downregulated by polyP, in turn, delaying tPA-mediated fibrinolysis. ${ }^{4}$ Here, we characterize the impact of polyP on uPA-mediated plasminogen activation. Our data reveal that polyP has a strong accelerating effect on uPA-mediated plasminogen activation that is highly dependent on concentration of polyP and plasminogen. Enhanced uPA-mediated plasminogen activation in the presence of polyP translates as a robust profibrinolytic effect in clot lysis assays. These data exemplify the complexity of polyP's action on hemostasis and illustrate that the presence of different activators and substrates in the local milieu can direct functional activity.

\section{Methods}

Additional experimental details can be found in the Online Supplementary Appendix.

\section{Ethical Consent}

Ethical approval was obtained from the University of Aberdeen College Ethics Review Board.

\section{Materials}

PolyP $\mathrm{P}_{00}$ was a kind gift from Dr Thomas Staffel BK Giulini GmbH (Ludwigshafen, Germany). PolyP 14, 60, 130 were a kind gift from Dr Toshikazu Shiba Regenetiss Inc. Medium chain (p100) polyP with and without biotin-labeling was from Kerafast Inc (Boston, MA, USA). PolyP concentrations are expressed as monomer concentrations throughout (monomer formula $\mathrm{NaPO}_{3}$ ). All reactions were carried out in TBST (50 mM Tris, $100 \mathrm{mM} \mathrm{NaCl}, 0.01 \%$ Tween-20, $\mathrm{pH}$ 7.4).

\section{Plasmin generation and uPA activity}

Purified human plasminogen-free fibrinogen $(2.4 \mu \mathrm{M})$, Glu- or Lys-plasminogen $(0-1 \mu \mathrm{M})$, tPA $(20 \mathrm{pM})$ or uPA $(180 \mathrm{pM}) \pm$ polyP $_{\text {es }}$ $(328 \mu \mathrm{M})$ in TBST was added in triplicate to 96 -well polystyrene plates. Clotting was initiated by thrombin $(0.25 \mathrm{U} / \mathrm{mL})$ and $\mathrm{CaCl}_{2}$ $(5 \mathrm{mM})$, and activity quantified using the fluorogenic substrate DVal-Leu-Lys 7-amido-4-methylcoumarin (D-VLK-AMC [0.35 $\mathrm{mM}]$ ) by measuring fluorescence release (excitation $360 / 40 \mathrm{~nm}$, emission $460 / 40 \mathrm{~nm}$ ) every minute ( $\mathrm{min}$ ) for 5 hours (h) in a Biotek Flx800 fluorescence microplate reader at $37^{\circ} \mathrm{C}$. The rate of plasmin generation was calculated using; Longstaff C, 2016, Shiny App for calculating zymogen activation rates, version 0.6 (https://drclongstaff.shinyapps.io/zymogenactnCL/).

UPA activity was determined by incubating the enzyme (180 pM) $\pm 328 \mu \mathrm{M}$ polyP with the chromogenic substrate CS-61 44 $(1.25 \mathrm{mM})$. Change in absorbance was measured every 30 seconds (s) at $405 \mathrm{~nm}$ for $200 \mathrm{~min}$.

\section{Protein binding assays to biotin-labelled polyP}

Binding of tPA, uPA, plasmin, FXII and activated FXII (FXIIa) to biotin-labelled polyP $(71 \mu \mathrm{M})$ were performed using an adaptation of the protocol described by Choi et al. ${ }^{13}$ Bound tPA, uPA or plasmin was detected with chromogenic substrates (1.2 mM S2288,

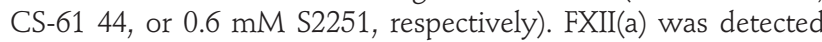
using a peroxidase conjugated goat anti-human FXII antibody.

\section{Turbidimetric fibrinolysis assays}

Purified human plasminogen-free fibrinogen $(2.4 \mu \mathrm{M})$, Glu- or Lys-plasminogen $(0-1 \mu \mathrm{M})$, tPA $(20 \mathrm{pM})$ or uPA $(180 \mathrm{pM})$ with or without polyP (0-1.3 $\mathrm{mM}$ ) in TBST was added in triplicate to 96well polystyrene plates. Clotting was initiated by thrombin $(0.25$ $\mathrm{U} / \mathrm{mL}$ ) and $\mathrm{CaCl}_{2}(5 \mathrm{mM})$, and turbidity monitored every min at $340 \mathrm{~nm}$ for $5 \mathrm{~h}$ at $37^{\circ} \mathrm{C}$ in a FLX-800 plate reader (Biotek Instruments).

\section{Confocal Microscopy}

Clots were prepared with human plasminogen-free fibrinogen $(2.65 \mu \mathrm{M})$ of which 9\% was DyLight 488-labeled, Glu-plasminogen $(1.25 \mu \mathrm{M})$ of which $20 \%$ was DyLight 633 -labeled, $\pm 328 \mu \mathrm{M}$ polyP in TBST in Ibidi -slides $\mathrm{VI}^{0.4}$. Thrombin $(0.25 \mathrm{U} / \mathrm{mL})$ and $\mathrm{CaCl}_{2}(5 \mathrm{mM})$ were added and fibrinolysis initiated by addition of tPA or uPA (75 nM).

Cascade blue ethylenediamine (CB)-labeled poly $\mathrm{P}_{0}$ was prepared as described. ${ }^{13}$ Clots were formed $\pm 328 \mu \mathrm{M}$ CB-polyP by polymerizing fibrinogen $(2.65 \mu \mathrm{M}$, with $9 \%$ labeled with DL550fibrinogen) as above.

\section{Statistical analysis}

Statistical analysis was performed in GraphPad Prism ${ }^{\circledR} 5.04$ using one-way analysis of variance or two-way analysis of variance with Bonferroni post hoc test or an unpaired Student's $t$-test (2tailed). $P<0.05$ was considered to be significant.

\section{Results}

\section{PolyP promotes uPA-mediated plasmin generation}

Our work has previously established that polyP interferes with the plasminogen activator function of $\mathrm{tPA}^{4}$ while augmenting plasminogen activation by FXIIa. ${ }^{14}$ Here we address the role of polyP on uPA-mediated plasminogen activation using the fluorogenic substrate D-VLKAMC. PolyP dramatically enhanced the rate of plasmin generation ( $3.96 \pm 0.34$ vs. $0.84 \pm 0.08 \mathrm{pM} / \mathrm{s} ; P<0.001)$ during uPA-mediated fibrinolysis (Figure 1A). In contrast, and consistent with our previous results 4 , a decrease in the rate $(0.74 \pm 0.06$ vs. $1.17 \pm 0.14 \mathrm{pM} / \mathrm{s}$ in $P<0.05)$ and amount of plasmin generation was observed during tPA-mediated fibrinolysis (Figure 1B). The ability of polyP to enhance the rate of uPA-mediated lysis was dose-dependent up to $32.8 \mu \mathrm{M}$ after which it decreased before increasing again at $164 \mu \mathrm{M}$ (Figure 1C). Above this concentration the rate of plasmin generation was greatly enhanced with a 6-fold increase at $328 \mu \mathrm{M}$ polyP. In marked contrast, downregulation of tPA-mediated plasmin generation required concentrations of greater than $70 \mu \mathrm{M}$ and was not strongly does-dependent (Figure 1D).

\section{Plasminogen concentration attenuates the cofactor function of polyP in uPA-mediated plasmin generation}

We next analysed the impact of plasminogen concentration on the cofactor function of polyP. Plasmin generation was monitored during clot lysis at various concentrations of plasminogen. The rate of plasmin generation by uPA and tPA in clots was directly proportional to the Glu-plas- 
minogen concentration (Figure 2A-B). The presence of polyP significantly increased the rate of uPA-mediated plasmin generation by around $75-82 \%$ at all Glu-plasminogen concentrations (Figure 2A). In marked contrast, polyP attenuated tPA-mediated plasmin generation at all Glu-plasminogen concentrations, with a maximal reduction at $1 \mu \mathrm{M}$ Glu-plasminogen (Figure $2 \mathrm{~B}$ ). Cleavage of the activation peptide of Glu-plasminogen by plasmin forms an intermediate form, Lys-plasminogen, which displays increased affinity for fibrin ${ }^{15}$ and is more readily cleaved by plasminogen activators. ${ }^{7,16,17}$ Overall plasmin generation by uPA occurs at a significantly faster rate in clots formed with Lys-plasminogen compared to Glu-plasminogen and is directly proportional to the concentration (Figure 2C-D). Interestingly, Lys-plasminogen overcame the cofactor function of polyP on uPA-mediated plasmin generation, significantly decreasing the magnitude of the effect from $79 \%$ at $0.125 \mu \mathrm{M}$ Lys-plasminogen to $9 \%$ at $1 \mu \mathrm{M}$ Lys-plasminogen (Figure 2C). With tPA there was a similar attenuation of plasmin generation (approximately $37 \%)$ at all Lys-plasminogen concentrations analysed (Figure 2B, D).

\section{polyP binds with a higher affinity to uPA than tPA}

We have previously demonstrated that polyP binds to Glu-plasminogen. ${ }^{14}$ Here we use biotin-labelled polyP in a plate-based assay to determine the binding affinity to (A) uPA and (B) tPA. polyP bound with approximately 25 -fold higher affinity to uPA $\left(K_{i}=9.8 \mathrm{nM}\right)$ than $\operatorname{tPA}\left(\mathrm{K}_{i}=245.3 \mathrm{nM}\right.$; Figure 3). Interestingly, binding of polyP did not alter the activity of uPA and no specific binding of polyP to plasmin was detected (data not shown). We have shown that polyP stimulates autoactivation of FXII to an active single chain
FXII (scFXIIa) ${ }^{18}$ and enhances the plasminogen activator capacity of FXIIa. ${ }^{14}$ We found that the high affinity interaction of UPA and polyP was similar to the interaction of polyP with FXII $\left(\mathrm{K}_{i}=7.9 \mathrm{nM}\right)$ and FXIIa $(\mathrm{Kd}=4.0 \mathrm{nM})$ (Online Supplementary Figure S1).

polyP enhances uPA-mediated lysis in a concentration and polymer length dependent manner

Incorporation of polyP during clot formation shortened the uPA-mediated $50 \%$ clot lysis time by $43.7 \pm 1.7 \mathrm{~min}$ $(P<0.0001$; Figure 4A). In contrast, tPA-mediated clot lysis was delayed on inclusion of polyP (Figure $4 \mathrm{~B}$ ), as previously reported. ${ }^{4}$ The cofactor function of polyP on uPA-mediated lysis was concentration dependent and required $66 \mu \mathrm{M}$ or higher to significantly stimulate the rate of lysis (Figure 4C). The use of polyP of more refined chain length revealed that polymers of $>14$-mer were required to promote uPA-mediated lysis (Figure 4D).

\section{Plasminogen modulates polyP-mediated effects on fibrinolysis}

The mode of action of tPA and uPA differ significantly, with tPA relying on the interaction with fibrin for efficient plasminogen activation, while uPA is fibrin independent. We investigated the relationship between plasminogen and polyP-mediated effects on fibrinolysis. A clear concentration dependence was observed with Glu-plasminogen on uPA-mediated lysis, with maximal lysis at $0.75 \mu \mathrm{M}$ plasminogen (Figure 5A). The impact of Glu-plasminogen on tPA-mediated lysis was less pronounced, but a small effect was observed with maximal lysis at $0.5 \mu \mathrm{M}$ plasminogen $(P<0.01$; Figure $5 \mathrm{~B})$. polyP significantly accelerated uPA-mediated lysis at all Glu-plasminogen concentra-
A

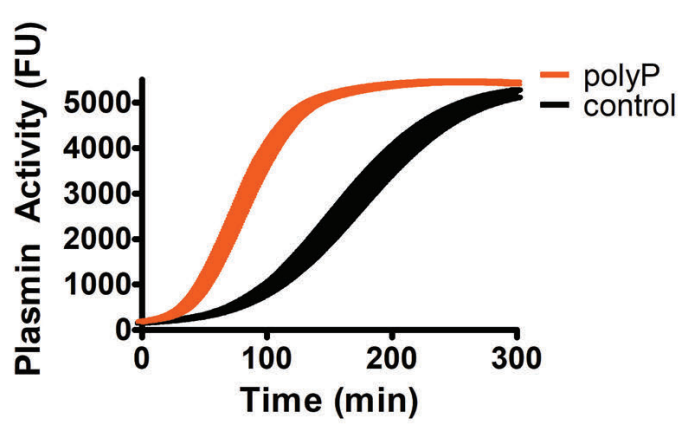

C

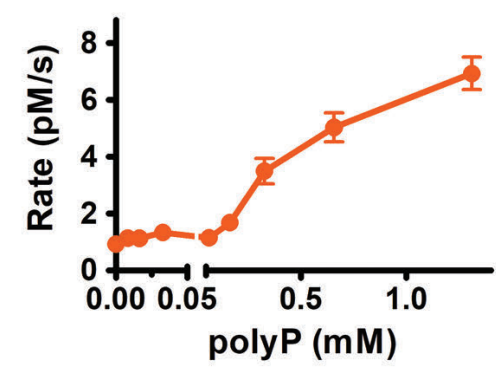

B

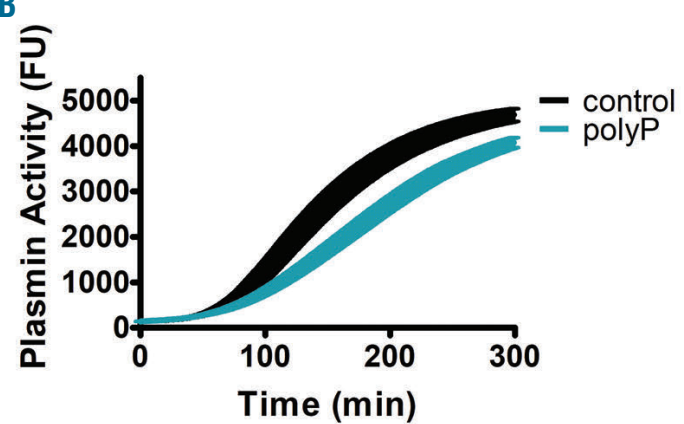

D

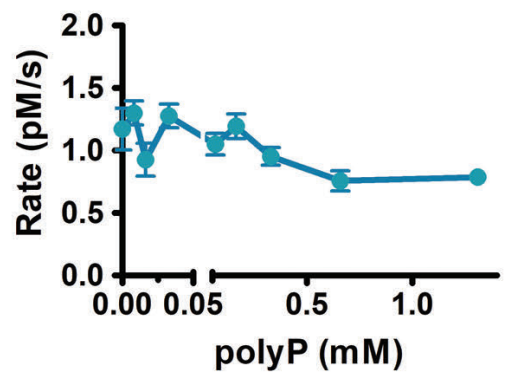

Figure 1. PolyP stimulates uPA-mediated plasmin generation in a concentration dependent manner. Fibrin clots were prepared containing $2.4 \mu \mathrm{M}$ fibrinogen, 0.24 $\mu \mathrm{M}$ Glu-plasminogen, $20 \mathrm{pM}$ tPA or $180 \mathrm{pM}$ uPA $\pm 328 \mu \mathrm{M}$ (polyP $\mathrm{F}_{\mathrm{s}}$. Clotting was initiated with thrombin $(0.25 \mathrm{U} / \mathrm{mL})$ and CaCl $(5 \mathrm{mM})$ and plasmin generation was quantified by incorporating the fluorogenic substrate D-VLK-AMC and monitoring fluorescence release (FU; Ex $360 \mathrm{~nm}$ Em $460 \mathrm{~nm}$ ). (A) uPA and (B) tPA mediated plasmin generation curves. Rate of plasmin generation in clots formed with varying polyP concentration (0-1.3 mM polyP $\left.\mathrm{P}_{\epsilon}\right)$ for (C) uPA and (D) tPA. Data are expressed as mean \pm standard error of the mean, $n \geq 3$. PolyP: polyphosphate; tPA: tissue plasminogen activator; uPA: urokinase plasminogen activator; FU: fluorescence units, Ex: excitation; EM: emission. 
tions tested, however, the cofactor function of polyP was tempered at high concentrations. Inclusion of polyP delayed tPA-mediated lysis at all Glu-plasminogen concentrations tested by an average of $40 \%$; therefore, increasing the Glu-plasminogen concentration did not alter the efficacy of polyP on tPA-driven lysis.

Clots containing Lys-plasminogen lyse significantly faster than those containing Glu-plasminogen (Figure 5C-
D). A dose-dependent relationship exists between Lys-plasminogen concentration and uPA-mediated lysis, with $50 \%$ lysis times decreasing from $51.1 \pm 1.36 \mathrm{~min}$ at $0.125 \mu \mathrm{M}$ to $17.2 \pm 0.32 \mathrm{~min}$ at $1 \mu \mathrm{M}$ (Figure $5 \mathrm{C}$ ). In contrast, there is no relationship between Lys-plasminogen concentration and tPA-mediated lysis, exemplifying the different modes of action of these plasminogen activators (Figure 5D). Similar to the results with plasmin generation,
A

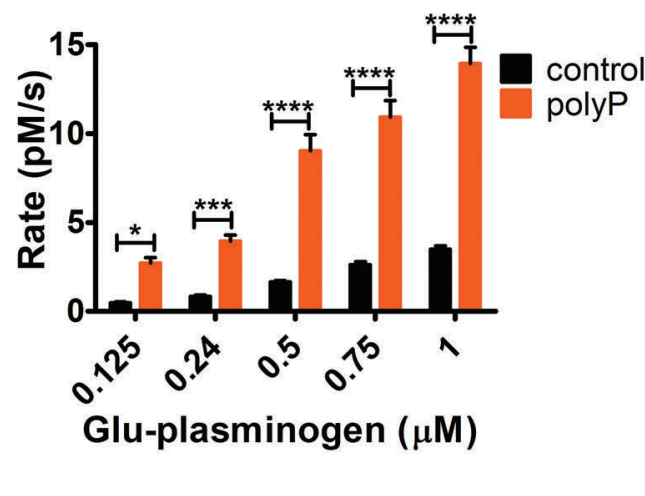

B

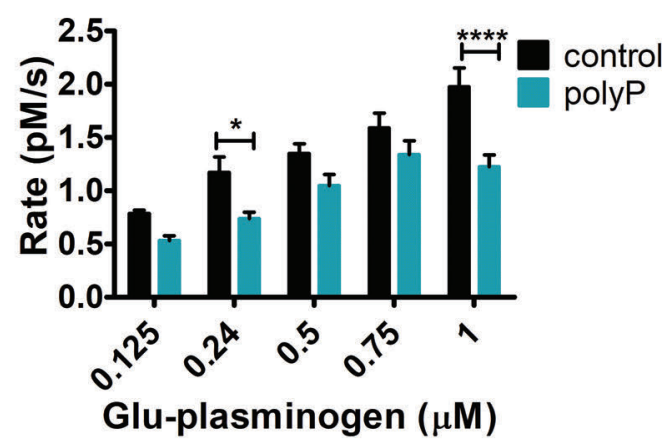

D

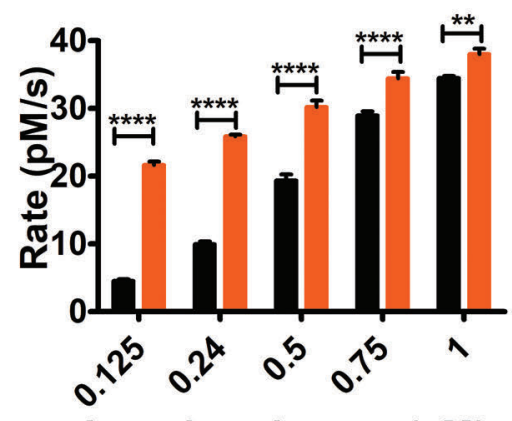

Lys-plasminogen $(\mu \mathrm{M})$

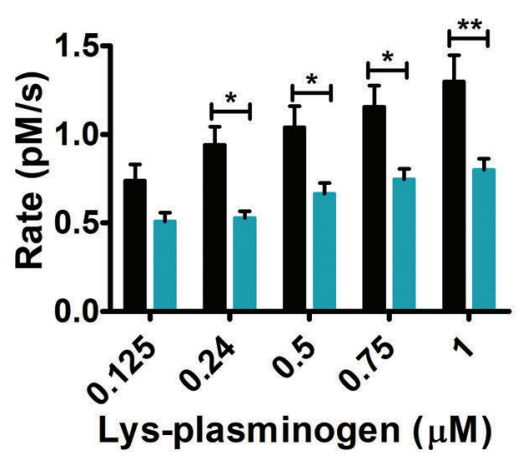

Figure 2. High concentrations of plasminogen attenuate the cofactor function of polyP in uPA-mediated plasminogen activation. Fibrin clots were prepared with 2.4 M fibrinogen, 0 -1 uM (A-B) Glu-plasminogen or (C-D) Lys-plasminogen, (A, C) $180 \mathrm{pM}$ uPA or (B, D) 20 pM tPA $\pm 328 \mu \mathrm{M}$ polyP $\mathrm{F}_{\mathrm{s} .}$. Clotting was initiated with thrombin $(0.25 \mathrm{U} / \mathrm{mL})$ and $\mathrm{CaCl}_{2}(5 \mathrm{mM})$. Plasmin generation in clots was quantified by incorporating the fluorogenic substrate D-VLK-AMC and monitoring fluorescence release (FU; Ex $360 \mathrm{~nm} \mathrm{Em} 460 \mathrm{~nm}$ ). $* P<0.05 ; * * P<0.01, * * * P<0.001$ and $* * * * P<0.0001$ compared with control clots. Data are expressed as mean \pm standard error of the mean, $n \geq 3$. polyP: polyphosphate; tPA: tissue plasminogen activator; uPA: urokinase plasminogen activator; FU: fluorescence units; Ex: excitation; EM: emission.

A

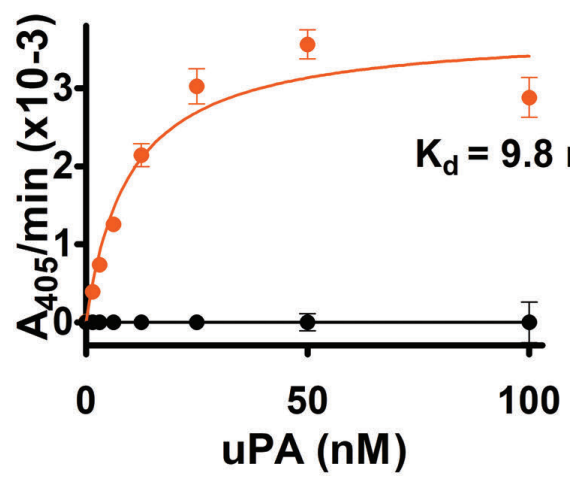

B

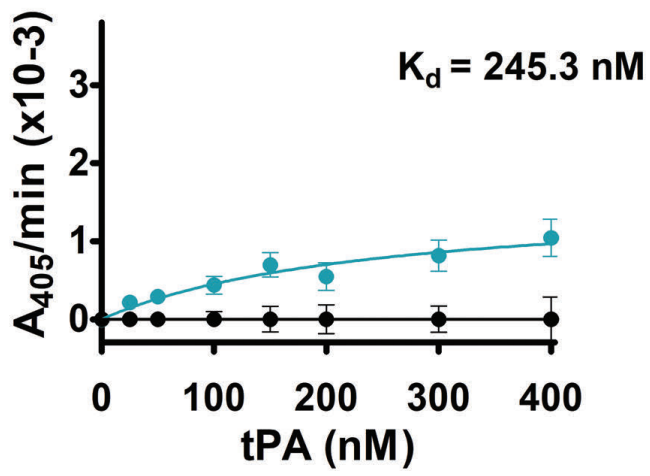

Figure 3. polyP binds to UPA with a significantly higher affinity than tPA. Binding of UPA or tPA (0-400 nM) to biotin-labelled polyP (71 $\mu \mathrm{M})$ bound to streptavidin coated stripwells. Bound UPA (orange) or tPA (blue) was detected with chromogenic substrates (S2288 or CS-61 44 respectively) by reading the change in absorbance at $405 \mathrm{~nm}$ every 30 seconds (s) for 200 minutes (min). No unspecific binding was detected in the absence of biotin-labelled polyP (black lines). Data are expressed as baseline corrected nonlinear fit as mean \pm standard error of the mean (SEM), $n=4$. PolyP: polyphosphate; tPA: tissue plasminogen activator; uPA: urokinase plasminogen activator. 
the cofactor function of polyP on uPA-mediated lysis is lost at high Lys-plasminogen concentrations (Figure 5C). Whereas, polyP delayed tPA-mediated lysis by approximately $20 \%$ at all Lys-plasminogen concentrations (Figure $5 \mathrm{~B}, \mathrm{D})$.

\section{polyP localization during fibrinolysis}

polyP is found to co-localize with fibrinogen, as previously reported, ${ }^{19}$ and plasminogen in fibrin clots. During fibrinolysis polyP concentrates within the fibrin dense knots of the clot alongside plasminogen and fibrinogen (Figure 6A). Colocalization was observed with both uPA and $\mathrm{tPA}$ but only the data for uPA is shown. Lysis was initiated at the edge of the clot, by addition of plasminogen activator, and as it progressed through the fibrin network polyP and plasminogen were found to remain colocalized within the lysis front (Figure 6B).

\section{Visualization of the cofactor function of polyP during uPA-mediated lysis in real-time}

Fluorescent confocal microscopy was used to visualize lysis in real-time following application of either uPA or tPA to the edge of fibrin clots. The rate of fibrinolysis in the control clot was similar in the presence of uPA or tPA (Figure 7A-B). As uPA-mediated lysis progresses plasmin(ogen) is dispersed throughout the clot, with a small concentrated zone at the leading edge (Figure 7A), in contrast plasminogen is restricted to a distinct zone at the leading edge in tPA-mediated lysis (Figure 7B). The incor- poration of polyP significantly accelerates uPA-mediated lysis (Figure 7A) with full lysis observed on average at 6 min (Online Supplementary Video S1B) compared to greater than $9 \mathrm{~min}$ in the control (Online Supplementary Video S1A).

\section{Discussion}

Over the last decade a role for the biomolecule polyP in the regulation of hemostasis has been exposed. The ability of polyP to modulate fibrinolysis manifests on several levels. We and others ${ }^{4,20}$ have demonstrated altered fibrin structure in the presence of polyP, a consequence of which is tempered binding of $\mathrm{tPA}$ and plasminogen to fibrin and subsequent down-regulation of fibrinolysis. ${ }^{4}$ In contrast, in this study we show that that polyP significantly accelerates uPA-mediated plasminogen activation thereby augmenting fibrinolysis. The rate of plasmin formation was approximately 5-fold faster in the presence of polyP and was dependent on concentration and polymer length, with polymers of 60 -mer or greater required to accelerate lysis. We determined that polyP binds with a 25 -fold higher affinity to uPA than tPA and potentially accelerates conversion of Glu-plasminogen to the intermediate form Lysplasminogen via a template mediated effect. Importantly, for the first time we visualize polyP during real-time lysis of fibrin clots and find it to be localized within fibrin dense areas alongside plasminogen, consistent with its known binding to these proteins. ${ }^{4,14,19}$
A

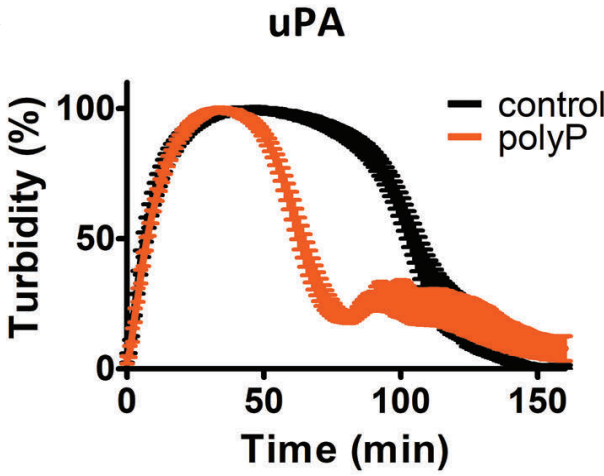

C

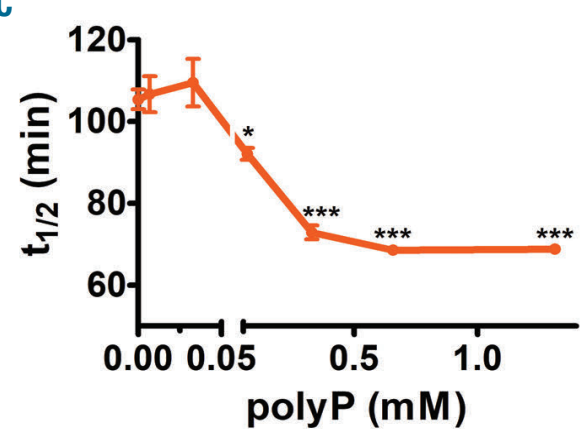

B

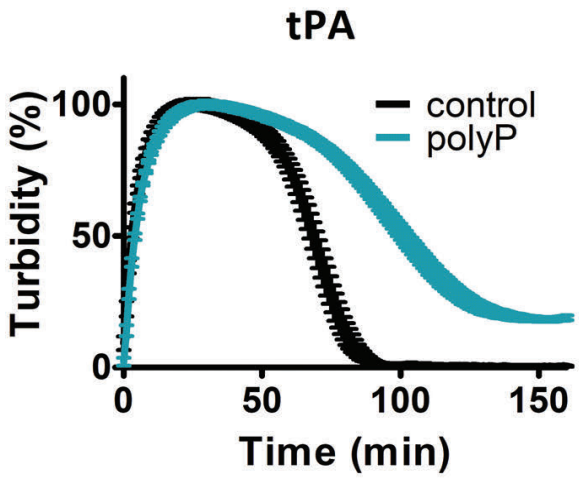

D

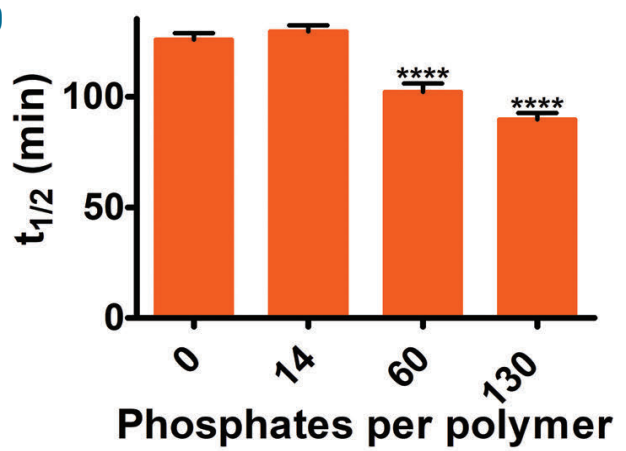

Figure 4. polyP acts as a cofactor to accelerate uPA-mediated fibrinolysis in a concentration and polymer size dependent manner. Fibrin clots were prepared containing $2.4 u \mathrm{M}$ fibrinogen, $0.24 \mu \mathrm{M}$ Glu-plasminogen, $20 \mathrm{pM}$ tPA or $180 \mathrm{pM}$ uPA $\pm 328 \mu \mathrm{M}$ polyP $\mathrm{P}_{\mathrm{se}}$. Clotting was initiated with thrombin $(0.25 \mathrm{U} / \mathrm{mL})$ and CaCl $(5 \mathrm{mM})$ and fibrinolysis monitored at $340 \mathrm{~nm}$ shown as percentage turbidity over time with (A) UPA or (B) tPA. (C) uPA-mediated fibrinolysis with 0-1.3 mM polyP or (D) polyP of various chain lengths at equivalent concentration of monomer $(328 \mu \mathrm{m})$. $* P<0.05, * * * P<0.001$ and $* * * * P<0.0001$ compared with control clots. Data are expressed as mean $50 \%$ lysis time $\left(\mathrm{t}_{1 / 2}\right) \pm$ standard error of the mean, $\mathrm{n} \geq 3$. polyP: polyphosphate; tPA: tissue plasminogen activator; uPA: urokinase plasminogen activator. 
A

$$
\text { UPA }
$$

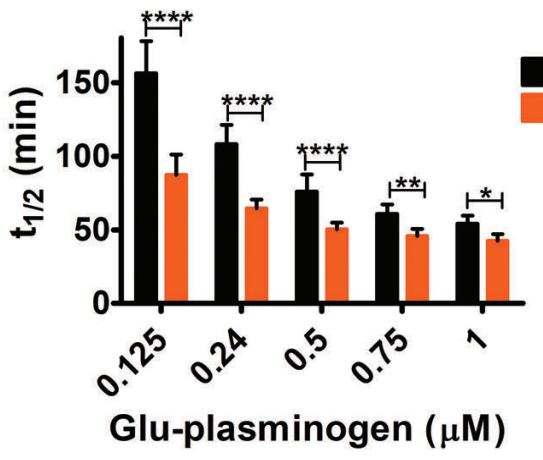

C

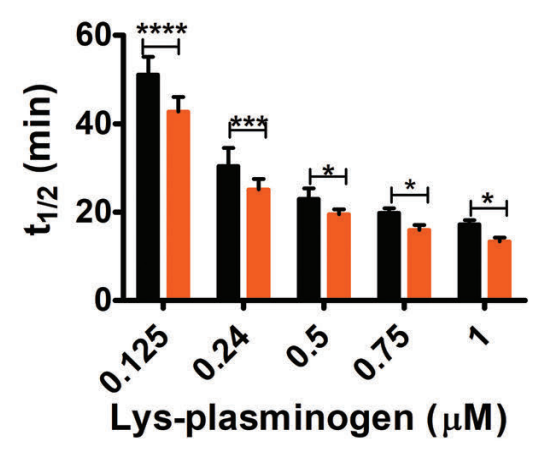

B

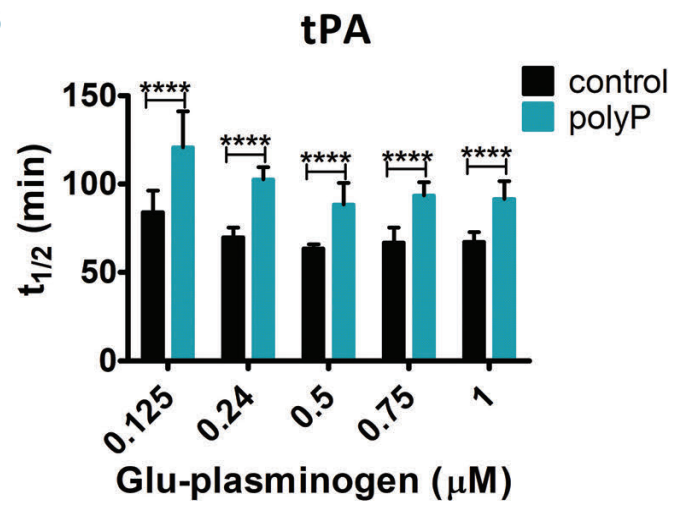

D

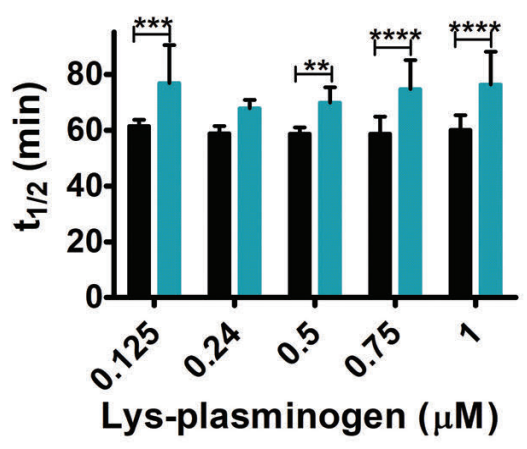

Figure 5. The cofactor function of polyP in uPA-mediated fibrinolysis is modulated by plasminogen concentration and form. Fibrin clots were prepared containing $2.4 \mu \mathrm{M}$ fibrinogen, 0-1 $\mu \mathrm{M}$ (A-B) Glu-plasminogen or (C-D) Lys-plasminogen, (A, C) $180 \mathrm{pM}$ uPA or (B, D) 20 pM tPA $\pm 328 \mu \mathrm{M}$ polyP $\mathrm{P}_{\text {ss. }}$ Clotting was initiated with thrombin $(0.25 \mathrm{U} / \mathrm{mL})$ and $\mathrm{CaCl}_{2}(5 \mathrm{mM})$ and fibrinolysis monitored at $340 \mathrm{~nm}$. $* P<0.05, * * P<0.01, * * * P<0.001$ and $* * * * P<0.0001$ compared with control clots. Data are expressed as mean \pm standard error of the mean, $n \geq 3$. polyP: polyphosphate; tPA: tissue plasminogen activator; uPA: urokinase plasminogen activator.

Despite their homology in structure, the mechanism of tPA and UPA-mediated plasminogen activation are very different. Efficient plasminogen activation by tPA requires its association with the fibrin surface, that is fibrin orchestrates its own destruction by plasmin. Binding of tPA to fibrin is largely attributed to its finger domain, ${ }^{21,22}$ while plasminogen associates with fibrin via kringle domains. ${ }^{23}$ The colocalization of enzyme and substrate on fibrin, as a surface, is crucial, as tPA is a poor plasminogen activator in solution. Circulating fibrinogen cannot accelerate plasminogen activation by tPA, as the sites are only exposed in fibrin ${ }^{24}$ thereby localizing plasmin formation to the fibrin clot. In marked contrast uPA does not bind fibrin and is reasonably efficient at activating plasminogen in solution. However, its cellular receptor, uPAR, localizes uPA via its amino terminal fragment to the cell surface, thereby augmenting plasminogen activation due to an increase in local reactant concentration.

The high affinity interaction of uPA and plasminogen ${ }^{14}$ with polyP and dependence on substrate and template concentration are indicative of a template mechanism of activation. In line with this polyP polymers of around 60mer were required to stimulate plasminogen activation by uPA, suggesting this length is critical to accommodate binding of both enzyme and substrate to the same template. Indeed, a template effect of polyP in thrombinmediated activation of FV to FVa has previously been reported. ${ }^{25}$ Polymers of around 80-100-mer are secreted following platelet stimulation ${ }^{2}$ with insoluble divalent cation bound polyP nanoparticles remaining associated with the activated platelet membrane. ${ }^{26}$ Platelet-derived polyP could actively participate in these template-mediated reactions within the milieu of a thrombus where local concentrations of the polymer will be high.

Previously an inhibitory effect of polyP on uPA-mediated fibrinolysis was described in a plasma based system. ${ }^{25}$ The data presented here delineates the role of polyP specifically on plasminogen activation by uPA using a purified system. The roles of polyP in hemostasis are multifaceted and coagulation and fibrinolysis are inextricably linked. It is therefore difficult, at this current time, to exclude the fact that these differences cannot be attributed to different experimental setups, reagents and indeed an impact of polyP on a different part of the pathway.

Intriguingly, the cofactor function of polyP described here in UPA-mediated plasminogen activation is markedly more pronounced than in uPA-mediated fibrinolysis. This can potentially be explained by an increase in the number of binding sites, that is plasminogen has the capacity to bind both polyP and lysine residues exposed on partially degraded fibrin generated following onset of lysis. ${ }^{23}$ Thus, the increase in surface binding sites on fibrin for plasminogen tempers the cofactor function of polyP. Therefore, there is a more evident effect at low plasminogen concentrations, where fewer lysine residues will be generated. Given the anionic nature of polyP it is feasible that it associates with positively charged lysine residues on fibrin, thereby obscuring the kringle-dependent binding of plasminogen to fibrin, however, high plasminogen concentrations eliminate the need for fibrin binding. 
A

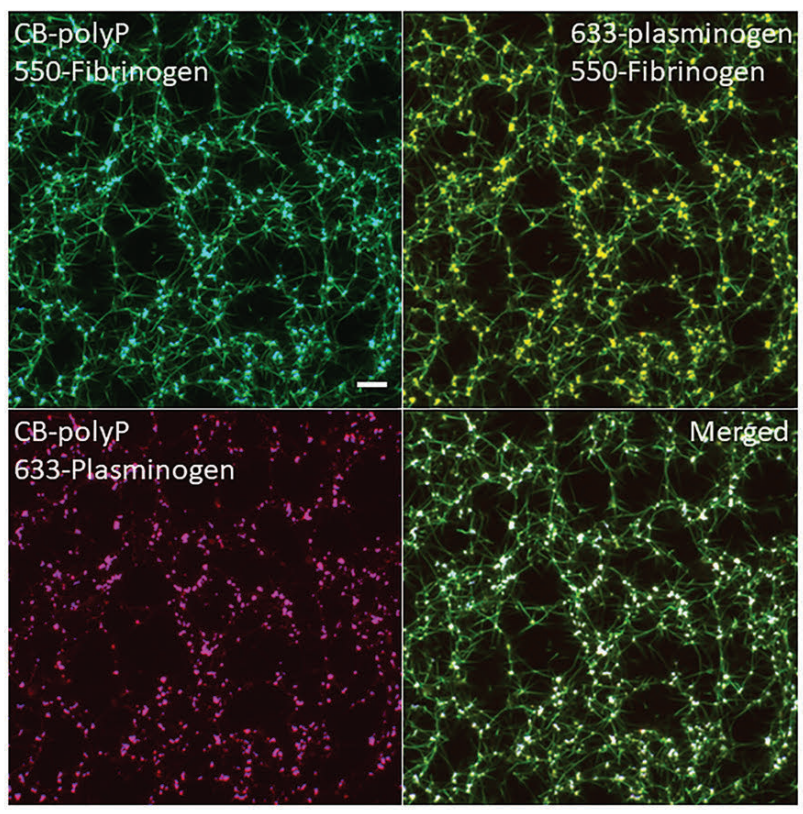

B

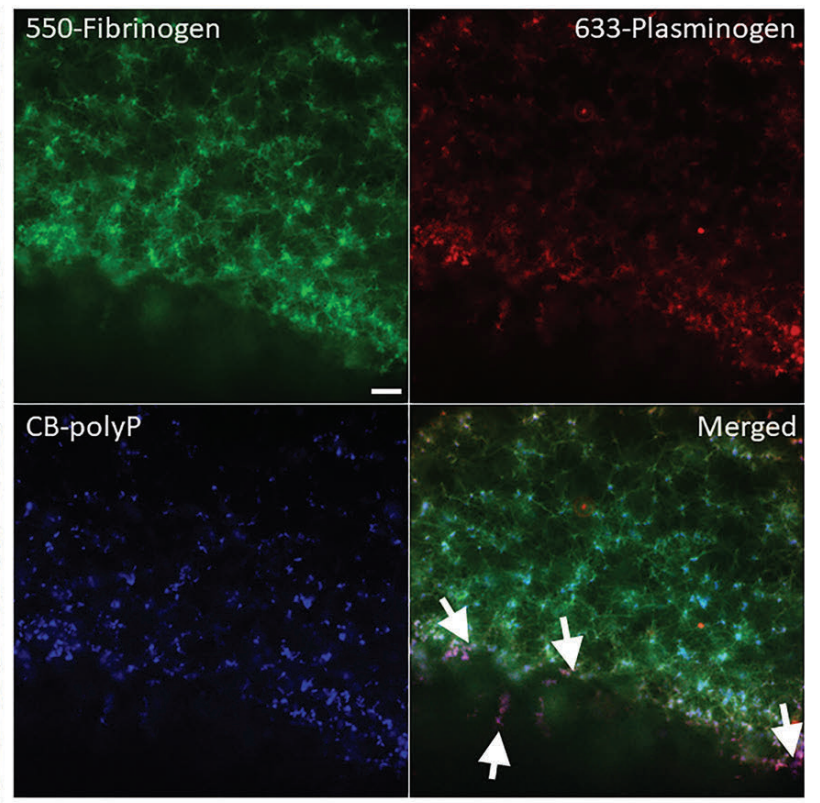

Figure 6. polyP colocalizes with fibrinogen and plasminogen during fibrinolysis. Fibrin clots were formed containing fibrinogen ( $2.65 \mu \mathrm{M}, 9 \%$ DyLight 550 -labeled), Glu-plasminogen (1.25 uM, 20\% DyLight 633-labeled) 328 uM Cascade-blue labeled polyP70 (CB-polyP), thrombin (0.25 U/mL), CaC minogen largely accumulate in the knotted regions of fibrin. (B) Fibrinolysis was initiated by exogenous uPA (75 nM). Plasminogen and polyP strongly colocalize at the lysis front during clot lysis, as indicated by the arrows. Scale bars $=10 \mu \mathrm{m}$. polyP: polyphosphate; uPA; urokinase plasminogen activator.

Our recent study ${ }^{19}$ investigating the molecular mechanisms underpinning the changes in fibrin structure in the presence of platelet-derived polyP revealed a significant change in fibrin polymerisation which stunts protofibril growth; thus providing an explanation for the characteristic 'knotted' appearance. ${ }^{4}$ Downstream this impacts on the mechanical properties of a clot, reducing overall stiffness and increasing its ability to deform plastically. ${ }^{19}$ Here, we find that polyP is localized within these 'knotted' regions of fibrin alongside plasminogen. Analysis of lysis in real-time reveals significant acceleration of uPA-mediated fibrinolysis presumably due to the direct colocalization of cofactor, enzyme and substrate. Previous work has shown that co-assembly of uPA and plasminogen on the same surface is not a prerequisite to stimulate plasmin formation. ${ }^{27}$ This crosstalk mechanism permits localization of plasminogen and uPA on different cellular surfaces or binding of plasminogen to fibrin while UPA is associated with cellular uPAR. A similar mechanism could explain our current observations; that is uPA is localized on polyP while plasminogen is bound to either polyP or partially degraded fibrin. Future work is necessary to ascertain the effect polyP may have on plasminogen activation on the surface of monocytes and neutrophils, which express both uPA and its cellular receptor UPAR.

Plasminogen circulates in the native or Glu-plasminogen form but can be cleaved by plasmin at Lys ${ }^{77}$-Lys ${ }^{78}$ to generate Lys-plasminogen. This cleavage prompts changes in the properties of the zymogen thereby providing a positive feedback mechanism. ${ }^{28}$ Lys-plasminogen is a considerably better substrate for both $\mathrm{tPA}^{29}$ and $\mathrm{uPA}^{30}$ and displays enhanced affinity for fibrin. ${ }^{31,32}$ Surface-bound Glu-plasminogen is more readily cleaved to Lys- plasminogen than in solution. ${ }^{33}$ We have previously shown, ${ }^{14}$ and confirmed in this study, that plasminogen, but not plasmin, bindsdi- rectly to polyP, as does the activator uPA. Co-assembly of Glu-plasminogen and uPA on the polyP surface will augment local concentrations of the reactants. An initial spark of plasmin formation will drive cleavage of Glu-plasminogen to Lys-plasminogen. Once formed Lys-plasminogen is more readily activated to plasmin than native Glu-plasminogen. Stimulation of uPA-mediated plasminogen activation by polyP is diminished when Lys-plasminogen is used, indicating that its cofactor function may lie in initial surface-mediated conversion of Glu- to Lys-plasminogen, ultimately accelerating plasmin formation. We have shown that plasmin does not bind to poly $\mathrm{P}^{4}$ or impact on its enzymatic activity indicating that the enhanced lysis arises at the level of plasminogen activation.

The lysine analogue, tranexamic acid (TXA), downregulates lysis by blocking tPA-mediated plasmin generation. The effect of TXA on uPA-mediated plasmin generation is more complex, with high concentrations of TXA augmenting plasmin generation, despite this fibrinolysis is still inhibited. Enhanced activation of plasminogen by uPA in the presence of TXA is indicative of a conformational change in plasminogen, from a closed structure to an open and more readily activated form. ${ }^{34}$ Our observations with polyP are similar, in that binding of Glu-plasminogen appears to augment its susceptibility to uPA-mediated cleavage indicative of a conformational change in the protein.

The fibrin specificity of tPA has led to the view that it is the dominant plasminogen activator in hemostasis, whereas, uPA has been implicated in plasmin-mediated cell migration, tissue remodelling and activation of latent growth factors and cytokines. ${ }^{35}$ TPA has been implicated in the degradation of deep vein thrombi in humans ${ }^{36}$ but genetic deficiency in mice has no impact. In marked contrast, uPA deficiency in mice markedly impairs venous thrombus resolution ${ }^{37}$ and conversely delivery of uPA to 
A

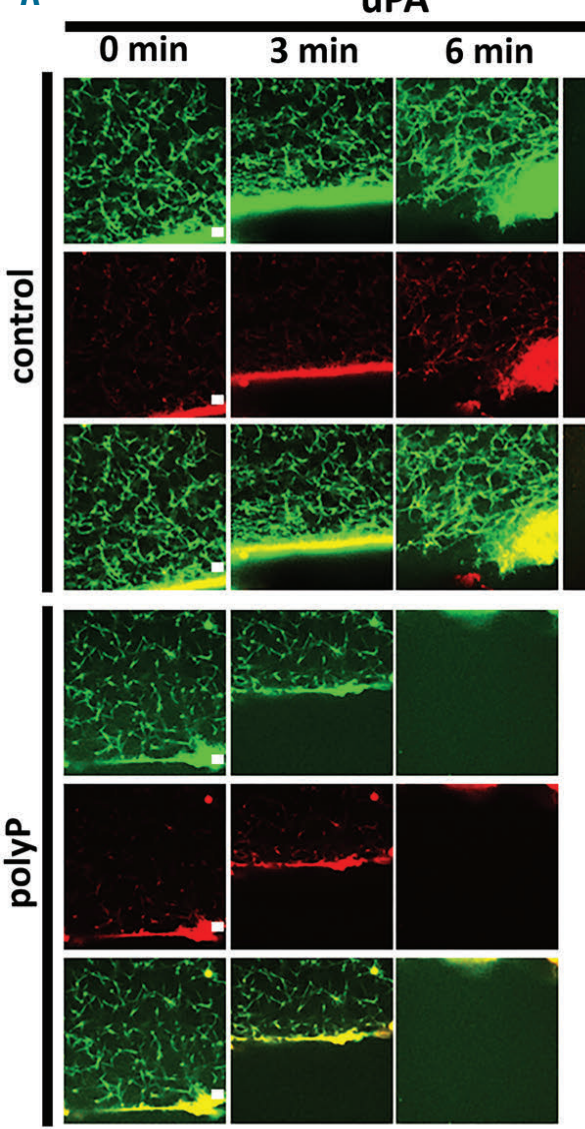

C

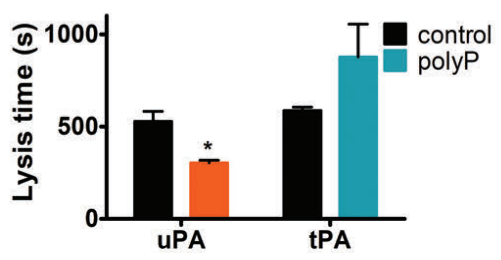

\section{$9 \min$}
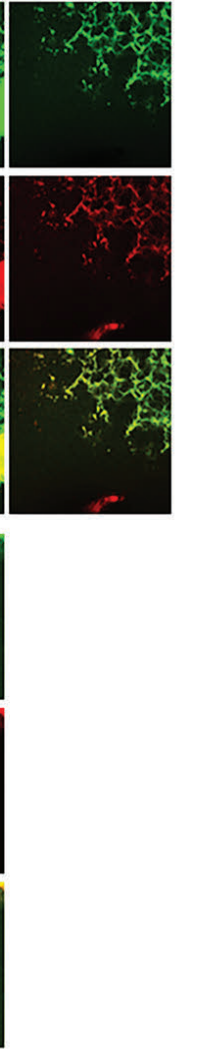

B

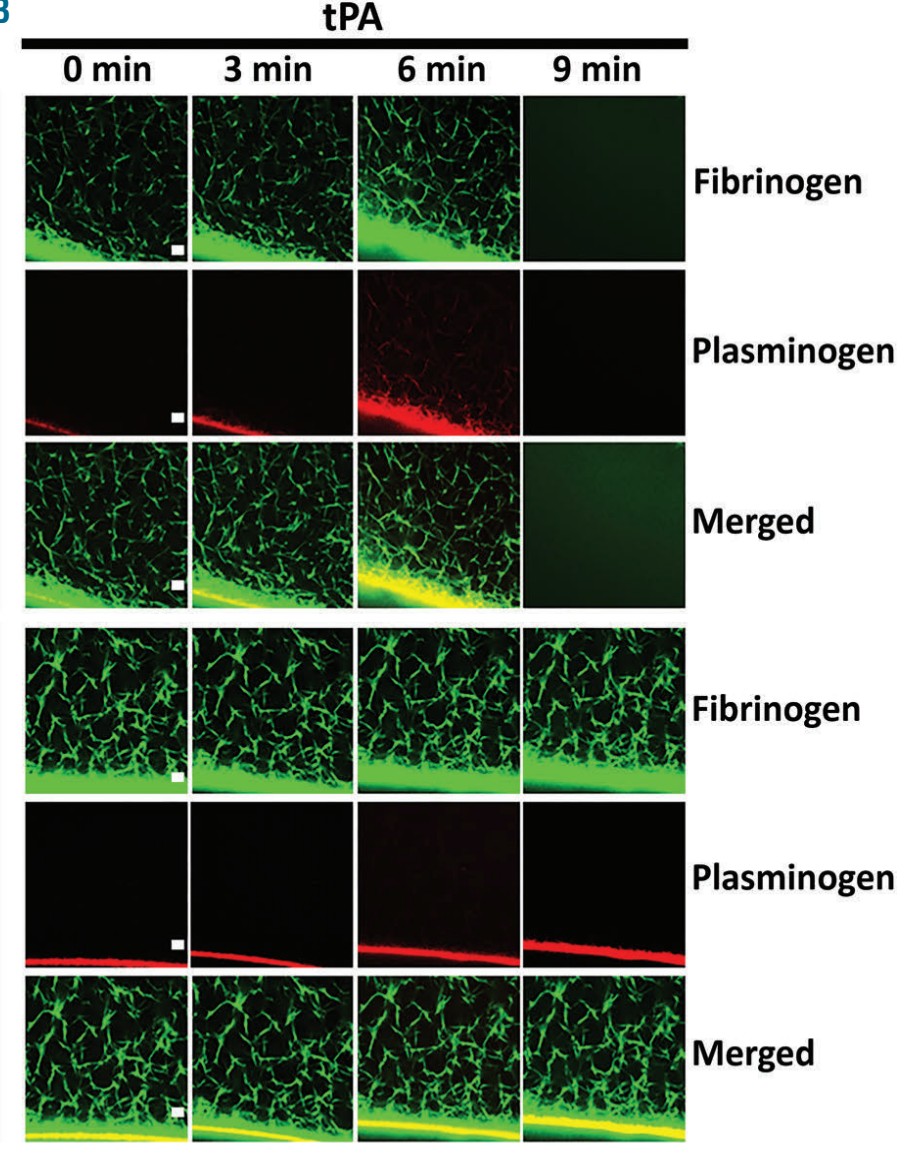

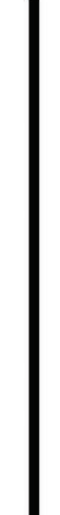

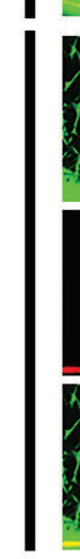

\begin{abstract}
Figure 7. Real-time lysis of fibrin clots reveals the cofactor function of polyP on uPA-mediated fibrinolysis. Fibrinolysis by exogenous PA $(75 \mathrm{nM})$ was monitored by fluorescent confocal microscopy of clots formed from fibrinogen (2.65 $\mu \mathrm{M}, 9 \%$ DyLight 488-labeled), plasminogen (1.25 $\mu \mathrm{M}, 20 \%$ DyLight 633-labelled), thrombin $(0.25 \mathrm{U} / \mathrm{mL}), \mathrm{CaCl}_{2}(5 \mathrm{mM}) \pm 328 \mu \mathrm{M}$ polyP $\mathrm{P}_{\omega}$. Images were taken every 15 seconds (s). (A) UPA and (B) tPA mediated fibrinolysis over time showing fibrinogen (green), plasminogen (red) and merged images where co-localisation is visualised as yellow. Representative image from $n=3$, scale bar $=10 \mu \mathrm{m}$. (C) Quantification of lysis time in $s$ as determined by the time taken to lyse scan area $(134.8 \mu \mathrm{m} \times 134.8 \mu \mathrm{m})$. Data expressed as mean \pm standard error of the mean, $n=3$. polyA: polyphosphate; tPA: tissue plasminogen activator; uPA: urokinase plasminogen activator.
\end{abstract}

thrombi, either directly ${ }^{38}$ or via uPA expressing monocytes, ${ }^{39}$ improves thrombus dissolution. Cross-talk between neutrophils, monocytes and platelets contributes to deep vein thrombosis ${ }^{40}$ and it is feasible that plateletderived polyP could augment monocyte-derived uPAmediated plasmin formation and facilitate thrombus resolution.

Platelets contain approximately $0.74 \mathrm{nmol} / 10^{8}$ platelets $^{1}$ therefore concentrations in whole blood are estimated to reach around $\approx 3 \mu \mathrm{M}$ following platelet activation. ${ }^{25}$ However, in platelet dense regions of thrombi concentrations could far exceed this; particularly as we and others have also shown that polyP remains bound to the activated platelet membrane. ${ }^{26,41}$ Elegant studies have revealed that solute transport within the core regions of thrombi is restricted $\mathrm{d}^{42-44}$ and as such the diffusion rate of plateletderived polyP within this milieu will be restricted. PolyP nanoparticles formed on the activated platelet membrane trigger contact activation ${ }^{26}$ and our laboratory has described a role for polyP in augmenting FXIIa-mediated plasminogen activation, with the platelet surface acting as a focal point for colocalization. ${ }^{14}$ By acting as a template for plasminogen activation by FXIIa or uPA, platelet-associated polyP nanoparticles could, under certain conditions, function to limit thrombus size.

Here we show that polyP binds to UPA with a high affinity and enhances uPA-mediated plasmin generation thereby augmenting the rate of fibrinolysis. This is in sharp contrast to the inhibitory effect of this polyanion on tPA-mediated plasminogen activation. ${ }^{4}$ The strong binding of uPA and plasminogen is suggestive of a template mechanism that results in enhanced conversion of Glu-plasminogen to Lys-plasminogen and is supported by the colocalization of the reactants on fibrin. The interaction of Glu-plasminogen with polyP may additionally result in a conformational change thereby facilitating its activation by uPA. Our data define a novel cofactor function of polyP in regulation of plasminogen activation by uPA that drives fibrinolysis in real time and may have important implications for physiological processes such as thrombus resolution, cell migration and tissue remodeling. 


\section{Disclosures}

No conflicts of interests to disclose.

\section{Contributions}

CSW performed the research, analysed the data and wrote the manuscript; NJM designed the research, analysed the data and wrote the manuscript.

\section{Acknowledgments}

We would like to thank Ms Michela Donnarumma and Ms
Linda Robertson for their invaluable technical assistance. We thank Dr Colin Longstaff, NIBSC, for invaluable advice on the kinetic assays and data analysis. We acknowledge the Microscopy and Histology Core Facility at the University of Aberdeen for excellent advice and use of the facilities.

\section{Funding}

This research was supported by grants FS/11/2/28579 (NJM) and $P G / 11 / 1 / 28461$ (NJM, CSW) from the British Heart Foundation.

\section{References}

1. Ruiz FA, Lea CR, Oldfield E, Docampo R. Human platelet dense granules contain polyphosphate and are similar to acidocalcisomes of bacteria and unicellular eukaryotes. J Biol Chem. 2004;279(43):44250-44257.

2. Muller F, Mutch NJ, Schenk WA, et al. Platelet polyphosphates are proinflammatory and procoagulant mediators in vivo. Cell. 2009;139(6):1143-1156.

3. Morrissey JH, Choi SH, Smith SA. Polyphosphate: an ancient molecule that links platelets, coagulation, and inflammation. Blood. 2012;119(25):5972-5979.

4. Mutch NJ, Engel R, Uitte de Willige S, Philippou H, Ariens RA. Polyphosphate modifies the fibrin network and down-regulates fibrinolysis by attenuating binding of tPA and plasminogen to fibrin. Blood. 2010;115(19):3980-3988

5. Robbins KC, Bernabe P, Arzadon L, Summaria L. NH2-terminal sequences of mammalian plasminogens and plasmin $\mathrm{S}$ carboxymethyl heavy (A) and light (B) chain derivatives. A re-evaluation of the mechanism of activation of plasminogen. J Biol Chem. 1973;248(20):7242-7246.

6. Mangel WF, Lin BH, Ramakrishnan V. Characterization of an extremely large, ligand-induced conformational change in plasminogen. Science. 1990;248(4951):69-73.

7. Sjoholm I. Studies on the conformational changes of plasminogen induced during activation to plasmin and by 6-aminohexanoic acid. Eur J Biochem. 1973;39(2):471-479.

8. Castellino FJ. Biochemistry of human plasminogen. Semin Thromb Hemost. 1984; 10(1):18-23.

9. Urano T, Chibber BA, Castellino FJ. The reciprocal effects of epsilon-aminohexanoic acid and chloride ion on the activation of human [Glu1]plasminogen by human urokinase. Proc Natl Acad Sci U S A. 1987; 84(12):4031-4034

10. Robbins KC, Summaria L, Hsieh B, Shah RJ. The peptide chains of human plasmin. Mechanism of activation of human plasminogen to plasmin. J Biol Chem. 1967; 242(10):2333-2342

11. Camiolo SM, Thorsen S, Astrup T. Fibrinogenolysis and fibrinolysis with tissue plasminogen activator, urokinase, streptokinase-activated human globulin, and plasmin. Proc Soc Exp Biol Med. 1971; 138(1):277-280

12. Stoppelli MP, Corti A, Soffientini A, Cassani G, Blasi F, Assoian RK. Differentiationenhanced binding of the amino-terminal fragment of human urokinase plasminogen activator to a specific receptor on U937 monocytes. Proc Natl Acad Sci U S A. 1985;
82(15):4939-4943.

13. Choi SH, Collins JN, Smith SA, DavisHarrison RL, Rienstra CM, Morrissey JH. Phosphoramidate end labeling of inorganic polyphosphates: facile manipulation of polyphosphate for investigating and modulating its biological activities. Biochemistry. 2010;49(45):9935-9941.

14. Mitchell JL, Lionikiene AS, Georgiev G, et al Polyphosphate colocalizes with factor XI on platelet-bound fibrin and augments its plasminogen activator activity. Blood. 2016; 128(24):2834-2845

15. Cockell CS, Marshall JM, Dawson KM, Cederholm-Williams SA, Ponting CP. Evidence that the conformation of unliganded human plasminogen is maintained via an intramolecular interaction between the lysine-binding site of kringle 5 and the N-terminal peptide. Biochem J. 1998;333 ( Pt 1):99-105.

16. Claeys H, Vermylen J. Physico-chemical and proenzyme properties of $\mathrm{NH} 2$-terminal glutamic acid and NH2-terminal lysine human plasminogen. Influence of 6-aminohexanoic acid. Biochim Biophys Acta. 1974; 342(2):351-359

17. Thorsen S, Mullertz S. Rate of activation and electrophoretic mobility of unmodified and partially degraded plasminogen. Effects of 6 aminohexanoic acid and related compounds. Scand J Clin Lab Invest. 1974; 34(2):167-176.

18. Engel R, Brain CM, Paget J, Lionikiene AS, Mutch NJ. Single-chain factor XII exhibits activity when complexed to polyphosphate. J Thromb Haemost. 2014;12(9):1513-1522.

19. Whyte CS, Chernysh IN, Domingues MM, et al. Polyphosphate delays fibrin polymerisation and alters the mechanical properties of the fibrin network. Thromb Haemost. 2016;116(5):897-903

20. Smith SA, Morrissey JH. Polyphosphate enhances fibrin clot structure. Blood. 2008; 112(7):2810-2816

21. van Zonneveld AJ, Veerman H, Pannekoek $\mathrm{H}$. On the interaction of the finger and the kringle-2 domain of tissue-type plasminogen activator with fibrin. Inhibition of kringle-2 binding to fibrin by epsilon-amino caproic acid. J Biol Chem. 1986; 261(30):14214-14218.

22. Longstaff C, Thelwell C, Williams SC, Silva MM, Szabo L, Kolev K. The interplay between tissue plasminogen activator domains and fibrin structures in the regulation of fibrinolysis: kinetic and microscopic studies. Blood. 2011;117(2):661-668.

23. de Vries C, Veerman H, Koornneef E, Pannekoek H. Tissue-type plasminogen activator and its substrate Glu-plasminogen share common binding sites in limited plasmin-digested fibrin. J Biol Chem. 1990; 265(23):13547-13552.
24. Medved L, Nieuwenhuizen W. Molecular mechanisms of initiation of fibrinolysis by fibrin. Thromb Haemost. 2003;89(3):409-419.

25. Smith SA, Mutch NJ, Baskar D, Rohloff P, Docampo R, Morrissey JH. Polyphosphate modulates blood coagulation and fibrinolysis. Proc Natl Acad Sci U S A. 2006; 103(4):903-908

26. Verhoef JJ, Barendrecht AD, Nickel KF, et al. Polyphosphate nanoparticles on the platelet surface trigger contact system activation. Blood. 2017;129(12):1707-1717.

27 Dejouvencel T, Doeuvre L, Lacroix R, et al Fibrinolytic cross-talk: a new mechanism for plasmin formation. Blood. 2010;115(10): 2048-2056.

28. Fredenburgh JC, Nesheim ME. Lys-plasminogen is a significant intermediate in the activation of Glu-plasminogen during fibrinolysis in vitro. J Biol Chem. 1992; 267(36):26150-26156.

29. Hoylaerts M, Rijken DC, Lijnen HR, Collen D. Kinetics of the activation of plasminogen by human tissue plasminogen activator. Role of fibrin. J Biol Chem. 1982;257(6) 2912-2919.

30. Wohl RC, Summaria L, Robbins KC Kinetics of activation of human plasminogen by different activator species at $\mathrm{pH} 7.4$ and 37 degrees C. J Biol Chem. 1980; 255(5):2005-2013.

31. Suenson E, Bjerrum P, Holm A, et al. The role of fragment $\mathrm{X}$ polymers in the fibrin enhancement of tissue plasminogen activator-catalyzed plasmin formation. I Biol Chem. 1990;265(36):22228-22237.

32. Nesheim M, Fredenburgh JC, Larsen GR The dissociation constants and stoichiometries of the interactions of Lys-plasminogen and chloromethyl ketone derivatives of tissue plasminogen activator and the variant delta FEIX with intact fibrin. J Biol Chem. 1990;265(35):21541-21548.

33. Miles LA, Castellino FJ, Gong Y. Critical role for conversion of glu-plasminogen to Lysplasminogen for optimal stimulation of plasminogen activation on cell surfaces. Trends Cardiovasc Med. 2003;13(1):21-30.

34. Silva MM, Thelwell C, Williams SC, Longstaff C. Regulation of fibrinolysis by Cterminal lysines operates through plasminogen and plasmin but not tissue-type plasminogen activator. J Thromb Haemost. 2012;10(11):2354-2360.

35. Mondino A, Blasi F. uPA and uPAR in fibrinolysis, immunity and pathology. Trends Immunol. 2004;25(8):450-455.

36. Killewich LA, Macko RF, Cox K, et al. Regression of proximal deep venous thrombosis is associated with fibrinolytic enhancement. J Vasc Surg. 1997;26(5):861-868.

37. Singh I, Burnand KG, Collins M, et al. Failure of thrombus to resolve in urokinase-type plasminogen activator gene-knockout mice: 
rescue by normal bone marrow-derived cells. Circulation. 2003;107(6):869-875

38. Gossage JA, Humphries J, Modarai B, Burnand KG, Smith A. Adenoviral urokinase-type plasminogen activator (uPA) gene transfer enhances venous thrombus resolution. J Vasc Surg. 2006;44(5):1085-1090.

39. Humphries J, Gossage JA, Modarai B, et al. Monocyte urokinase-type plasminogen activator up-regulation reduces thrombus size in a model of venous thrombosis. J Vasc Surg. 2009;50(5):1127-1134.

40. von Bruhl ML, Stark K, Steinhart A, et al.
Monocytes, neutrophils, and platelets cooperate to initiate and propagate venous thrombosis in mice in vivo. J Exp Med. 2012; 209(4):819-835

41. Mitchell JL, Lionikiene AS, Georgiev G, et al. Polyphosphate co-localizes with factor XII on platelet-bound fibrin and augments its plasminogen activator activity. Blood. 2016; 128(24):2834-2845

42. Welsh JD, Stalker TJ, Voronov R, et al. A systems approach to hemostasis: 1 . The interdependence of thrombus architecture and agonist movements in the gaps between platelets. Blood. 2014;124(11):1808-1815.

43. Tomaiuolo M, Stalker TJ, Welsh JD, Diamond SL, Sinno T, Brass LF. A systems approach to hemostasis: 2 . Computational analysis of molecular transport in the thrombus microenvironment. Blood. 2014 124(11):1816-1823.

44. Stalker TJ, Welsh JD, Tomaiuolo M, et al. A systems approach to hemostasis: 3 . Thrombus consolidation regulates intrathrombus solute transport and local thrombin activity. Blood. 2014; 124(11): 1824-1831. 